\title{
Comportamento do Tebuthiuron em Solo de Cultivo de Cana-de- AÇÚCAR UTILIZANDo LISÍMETRO DE DRENAGEM MOdIFICAdo ${ }^{1}$
}

\author{
Tebuthiuron Behavior In Sugar Cane Soil Using Modified Drainage Lysimeter
}

\author{
SOUZA, E.L.C. ${ }^{2}$, FOLONI, L.L. ${ }^{3}$, MANTOVANI, E.C. ${ }^{4}$ e TEIXEIRA FILHO, J. ${ }^{5}$
}

\begin{abstract}
RESUMO - O tebuthiuron é um dos herbicidas mais usados no plantio de cana-de-açúcar, no Estado de São Paulo. Estudos têm sido realizados para determinar o índice de lixiviação do tebuthiuron e monitorar sua presença nos mananciais de águas superficiais e subterrâneas, ainda sem uma conclusão definitiva. Com o objetivo de avaliar, em condições de campo, a movimentação vertical do herbicida tebuthiuron em Latossolo Vermelho distroférrico de textura argilosa, testou-se, em ambiente controlado, a hipótese de que o tebuthiuron apresenta baixa mobilidade vertical e pequeno potencial de contaminação de águas subterrâneas. O trabalho foi conduzido no Campo Experimental da Faculdade de Engenharia Agricola da Unicamp, utilizando-se lisimetro de drenagem modificado de $2 \mathrm{~m}$ de diâmetro e $3 \mathrm{~m}$ de profundidade, com dez pontos verticais, por meio dos quais foram coletadas amostras de água da chuva. As amostras foram submetidas à Análise Cromatográfica Líquida de Alta Eficiência. Os dados obtidos indicaram presença decrescente do herbicida nas amostras coletadas no período de março a agosto de 2006: amostra 1 - 0,020 g i.a. (5,3\%); 2 $-0,016$ g i.a. $(4,3 \%) ; 3-0,015$ g i.a. $(4,0 \%) ; 4-0,014$ g i.a. $(3,7 \%) ; 5-0,014$ g i.a. $(3,7 \%) ; 6-$ 0,007 g i.a. $(1,9 \%) ; 7-0,002$ g i.a. $(0,5 \%)$; e 8 - 0,001 g i.a. (0,3\%) do total aplicado na área do lisimetro $(0,3768 \mathrm{~g}$ i.a.), confirmando a hipótese de baixa mobilidade vertical do tebuthiuron em Latossolo Vermelho distroférrico de textura argilosa, indicando, para esse solo, pequeno potencial de contaminação das águas subterrâneas.
\end{abstract}

Palavras-chave: herbicida, lixiviação, movimentação vertical, águas subterrâneas.

\begin{abstract}
Tebuthiuron is one of herbicides most used on sugar cane in the state of São Paulo. Studies have been carried out to determine tebuthiuron leaching index and to monitor its presence in surface and groundwater sources, with no definitive conclusion been reached yet. The aim of this research was to evaluate the vertical movement of tebuthiuron under field conditions on Clayey Dystroferric Red Soil. The tested hypothesis was based on the low vertical mobility and small potential of groundwater contamination by tebuthiuron. The trial was carried out at the Experimental Field College of Agricultural Engineering - UNICAMP, using a modified drainage lysimeter, 2 meters of diameter and 3 meters depth, with 10 vertical points where rain water samples were collected. The samples were submitted by High Performance Liquid Chromatography (HPLC). The data found indicated a decreasing presence of the herbicide in the samples collected through time - March to August, 2006: sample $1-0.020 \mathrm{~g}$ a.i. (5.3\%); $2-0.016 \mathrm{~g}$ a.i. (4.3\%); 3 - $0.015 \mathrm{~g}$ a.i. (4.0\%); 4 - $0.014 \mathrm{~g}$ a.i. (3.7\%); 5 - $0.014 \mathrm{~g}$ a.i. (3.7\%); $6-0.007 \mathrm{~g}$ a.i. (1.9\%); 7 - $0.002 \mathrm{~g}$ a.i. (0.5\%); 8 $-0.001 \mathrm{~g}$ a.i. $(0.3 \%)$-total applied in the lysimeter area (0.3768 $\mathrm{g}$ i.a.), confirming the hypothesis that tebuthiuron vertical mobility is low in Clayey Dystroferric Red Soil, with clayey texture, indicating small potential of groundwater contamination on this soil.
\end{abstract}

Keywords: herbicide, leaching, vertical mobility, groundwater.

Recebido para publicação em 2.7.2007 e na forma revisada em 27.2.2008.

Engo-Agrọ , M.S., Programa de Pós-Graduação em Engenharia Agrícola, FEAGRI/UNICAMP, <elcorrea77@hotmail.com>, ${ }^{3}$ Engô-Agr ${ }^{0}$, Prof. Dr., colaborador do curso de Pós-Graduação em Engenharia Agrícola, FEAGRI/UNICAMP, Campinas-SP, ${ }^{4}$ Matemático, M.S., aluno de Pós-Graduação em Engenharia Agrícola, FEAGRI/UNICAMP, Campinas-SP; ${ }^{5}$ Eng. Civil, Prof. Dr. da Faculdade de Engenharia Agrícola, FEAGRI/UNICAMP, Campinas-SP. 


\section{INTRODUÇÃO}

A atividade agrícola tradicional, que visa aumentar os indices de produtividade, requer uso de quantidade considerável de defensivos agrícolas (Spadotto et al., 2004). Dentre eles, os herbicidas representam a maior parcela do mercado em todos os países do mundo. No Brasil, os herbicidas correspondem a $56 \%$ do volume dos defensivos agrícolas comercializados. Como, no País, a cultura da cana-de-açúcar é a segunda em consumo desses defensivos agrícolas (Procópio et al., 2003) e o tebuthiuron é um dos herbicidas mais intensamente utilizados nessa cultura (Gomes et al., 2006), o uso desse produto tem despertado preocupação em relação ao risco ambiental, principalmente no tocante à contaminação dos lençóis freáticos.

Alguns trabalhos, a partir de critérios teóricos e modelos matemáticos (Pessoa et al., 2003; Duarte, 2003; Inoue et al., 2003; Luiz et al., 2004; Armas et al., 2005; Lourencetti et al., 2005), descrevem a suscetibilidade da água subterrânea à contaminação pelo herbicida tebuthiuron. Por outro lado, pesquisas realizadas em laboratório e em campo por Lorenzi (1984), Blanco \& Lorenzi (1984), Matallo et al. (2003), Boeira \& Dornelas de Souza (2005), Matallo et al. (2005), Gomes et al. (2006) e Cerdeira et al. (2006), utilizando colunas de solo e coletas de amostras de solo e água, apontam menor lixiviação deste herbicida. Há, portanto, dúvidas quanto ao seu potencial de contaminação.

Com o objetivo de avaliar, em condições de campo, a possivel movimentação vertical do herbicida tebuthiuron em Latossolo Vermelho distroférrico de textura argilosa, testou-se, por meio de Lisímetro de Drenagem Modificado, a hipótese de que o tebuthiuron apresenta baixa mobilidade vertical e pequeno potencial de contaminação de águas subterrâneas em solo de textura característica para cultivo de cana-de-açúcar no Estado de São Paulo.

\section{MATERIAL E MÉTODOS}

O experimento foi conduzido no Campo Experimental da Faculdade de Engenharia Agrícola da Unicamp, em Latossolo Vermelho distroférrico de textura argilosa (Embrapa,
1999; Oliveira et al., 1999). O clima da região é uma transição entre os tipos Cwa e Cfa, de acordo com classificação de Köppen, sendo considerado tropical de altitude com inverno seco e verão úmido. A temperatura do mês mais quente (fevereiro) é superior a $22{ }^{\circ} \mathrm{C}$, podendo atingir $36^{\circ} \mathrm{C}$. A precipitação média anual é $1.382 \mathrm{~mm}$, sendo o periodo chuvoso de outubro a março (1.048 $\mathrm{mm})$, quando ocorre $75 \%$ do total de chuva anual. O periodo mais seco ocorre de junho a setembro.

Amostras de solo foram coletadas e submetidas à análise pelo Instituto Agronômico de Campinas (IAC), para determinação das propriedades físico-químicas do solo (densidade, $\%$ areia, textura, matéria orgânica, $\mathrm{pH}$, soma de bases, CTC, V\%, condutividade hidráulica), que estão descritas nas Tabelas 1, 2 e 3.

O lisimetro de drenagem modificado, montado no experimento, é um modelo adaptado de experiência realizada por Weber \& Keller (Leng et al., 2000). É constituído por uma coluna de solo indeformado com $2,0 \mathrm{~m}$ de diâmetro e 3,0 m de profundidade. A construção do lisímetro de drenagem modificado (Figuras 1 e 2) obedeceu às etapas a seguir. Demarcou-se uma área no campo e, nela, um círculo de $2 \mathrm{~m}$ de diâmetro; a partir da borda do círculo, abriuse um fosso circular de $1 \mathrm{~m}$ de largura por $3 \mathrm{~m}$ de profundidade, demarcando um cilindro com as medidas citadas, mantendo-se as características físicas do solo. Impermeabilizou-se o cilindro com plástico de cor preta e espessura de $2 \mathrm{~mm}$. Devolveu-se o solo retirado ao redor do cilindro, recuperando-se o espaço original. Abriu-se uma trincheira com 0,9 m de largura, 2,20 m de comprimento e 3,0 m de profundidade, a uma distância de $0,85 \mathrm{~m}$ da borda do cilindro. Prepararam-se coletores de água constituídos por dez tubos de PVC branco de $1 \frac{1}{2}$ polegada e $1,5 \mathrm{~m}$ de comprimento, com corte longitudinal de $40 \mathrm{~cm}$ de comprimento numa das pontas, formando uma canaleta com a metade do diâmetro do tubo, para funcionar como coletor d'água; na outra ponta do tubo foi feita uma rosca para colocação de tampa a ser aberta, para coleta das amostras. Na parede da trincheira, foram abertas, com auxílio de trado, dez perfurações eqüidistantes em sentido diagonal, mantendo um aclive de 5\%. Os tubos preparados foram inseridos nessas perfurações com as canaletas posicionadas dentro do 
Tabela 1 - Descrição dos horizontes, porcentagem de areia, silte e argila e classificação textural do perfil do solo

\begin{tabular}{|c|c|c|c|c|c|c|}
\hline Horizonte & $\begin{array}{c}\text { Profundidade } \\
(\mathrm{cm})\end{array}$ & Areia grossa & Areia fina & Silte & Argila & $\begin{array}{c}\text { Classificação } \\
\text { textural }\end{array}$ \\
\hline $\mathrm{Ap}$ & $0-18$ & 14,8 & 12,2 & 11,6 & 61,5 & Muito argilosa \\
\hline $\mathrm{AB}$ & $18-40$ & 15,3 & 12,3 & 12,3 & 60,1 & Muito argilosa \\
\hline $\mathrm{Bt} 1$ & $40-75$ & 12,9 & 10,7 & 5,8 & 70,6 & Muito argilosa \\
\hline $\mathrm{Bt} 2$ & $75-110$ & 11,8 & 10,1 & 6,3 & 71,9 & Muito argilosa \\
\hline $\mathrm{Bw} 1$ & $110-200$ & 13,4 & 11,5 & 5,4 & 69,8 & Muito argilosa \\
\hline $\mathrm{Bw} 2$ & $200-300$ & 13,8 & 11,7 & 6,0 & 68,6 & Muito argilosa \\
\hline
\end{tabular}

Tabela 2 - Descrição da microporosidade, macroporosidade, porosidade total e densidade do perfil do solo

\begin{tabular}{|c|c|c|c|c|}
\hline Horizonte & $\begin{array}{c}\text { Microporosidade } \\
\left(\mathrm{m}^{3} \cdot \mathrm{m}^{3}\right)\end{array}$ & $\begin{array}{c}\text { Macroporosidade } \\
\left(\mathrm{m}^{3} \cdot \mathrm{m}^{3}\right)\end{array}$ & $\begin{array}{c}\text { Porosidade total } \\
\left(\mathrm{m}^{3} \cdot \mathrm{m}^{3}\right)\end{array}$ & $\begin{array}{c}\text { Densidade } \\
\left(\mathrm{mg} \cdot \mathrm{m}^{3}\right)\end{array}$ \\
\hline $\mathrm{Ap}$ & 0,32 & 0,42 & 0,74 & 0,86 \\
\hline $\mathrm{AB}$ & 0,20 & 0,41 & 0,61 & 1,03 \\
\hline $\mathrm{Bt} 1$ & 0,23 & 0,38 & 0,61 & 0,85 \\
\hline $\mathrm{Bt} 2$ & 0,18 & 0,39 & 0,57 & 0,90 \\
\hline Bw1 & 0,20 & 0,40 & 0,60 & 0,74 \\
\hline Bw2 & 0,19 & 0,41 & 0,60 & 0,79 \\
\hline
\end{tabular}

Tabela 3 - Descrição das características químicas do perfil do solo

\begin{tabular}{|c|c|c|c|c|c|c|}
\hline Horizonte & $\mathrm{MO}\left(\mathrm{g} \cdot \mathrm{dm}^{-3}\right)$ & $\mathrm{pH}$ & $\mathrm{SB}$ & $\mathrm{CTC}$ & $\mathrm{V} \%$ & $\mathrm{~K}_{0}(\mathrm{~m} \mathrm{~m} / \mathrm{h})$ \\
\hline $\mathrm{Ap}$ & 41 & 5,3 & 40,0 & 78,1 & 51 & 469,02 \\
\hline $\mathrm{AB}$ & 33 & 4,9 & 19,5 & 66,5 & 29 & 48,15 \\
\hline $\mathrm{Bt} 1$ & 21 & 5,2 & 26,3 & 57,1 & 46 & 52,97 \\
\hline $\mathrm{Bt} 2$ & 16 & 5,4 & 20,1 & 45,1 & 45 & 130,36 \\
\hline Bw1 & 13 & 5,8 & 15,0 & 35,2 & 43 & 103,70 \\
\hline Bw2 & 11 & 6,1 & 16,3 & 32,7 & 50 & 138,80 \\
\hline
\end{tabular}

cilindro. As pontas com as roscas ficaram a $10 \mathrm{~cm}$ da parede da trincheira; em volta do conjunto trincheira e cilindro foi construída uma proteção retangular em concreto de 5,70 x 2,70 m; e em volta do cilindro inseriu-se uma chapa metálica, para garantia de que este recebesse somente água da chuva.

O plantio da cana-de-açúcar foi realizado em 15 de fevereiro de 2006 (Figura 3), após construção do lisímetro, em profundidade média de 40-50 cm, utilizando o cultivar RB835486 , conforme as recomendações técnicas de
Coleti (1987). Em seguida, aplicou-se o tebuthiuron, na forma de suspensão concentrada, $500 \mathrm{~g} \mathrm{~L}^{-1}$.

Considerou-se a maior dose recomendada para a cultura - 2,4 $\mathrm{L} \mathrm{ha}^{-1}$ ou $1,2 \mathrm{~kg} \mathrm{ha}^{-1} \mathrm{de}$ ingrediente ativo (i.a.) -, conforme recomendação do fabricante (Dow AgroSciences, 2005). A aplicação foi efetuada em 3 de março de 2006, utilizando-se equipamento de precisão a gás carbônico (CO2) - R\&D Sprayers - provido de barra compensada com quatro bicos Teejet XR 110.03, jato plano, espaçados de 


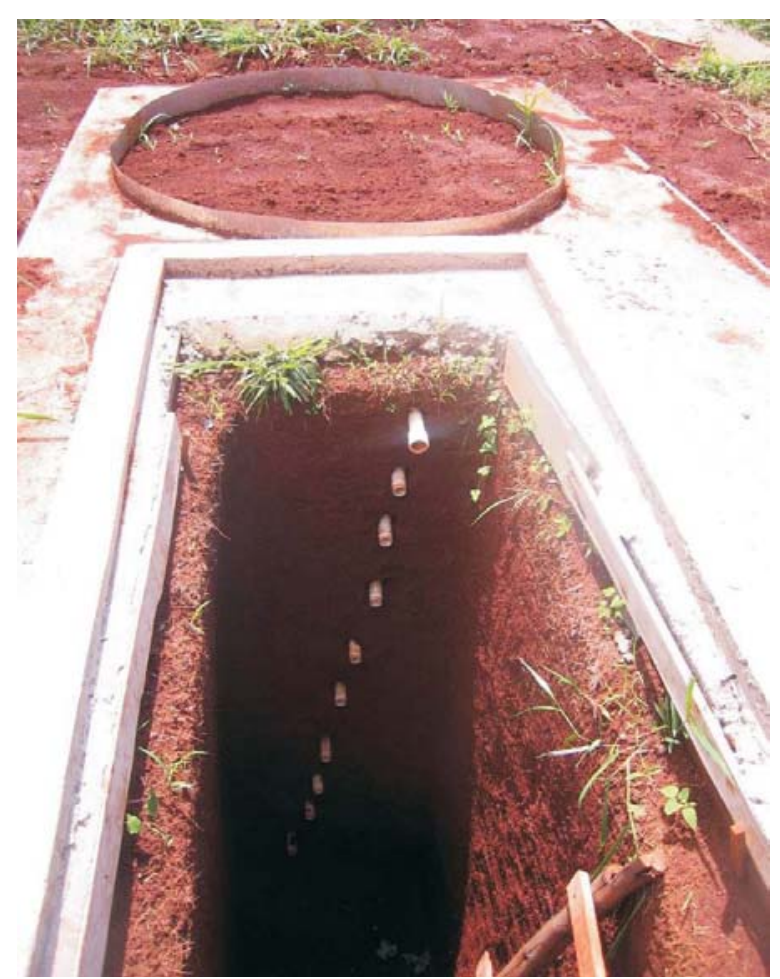

Figura 1 - Lisímetro montado no campo experimental da FEAGRI (fevereiro 2006).

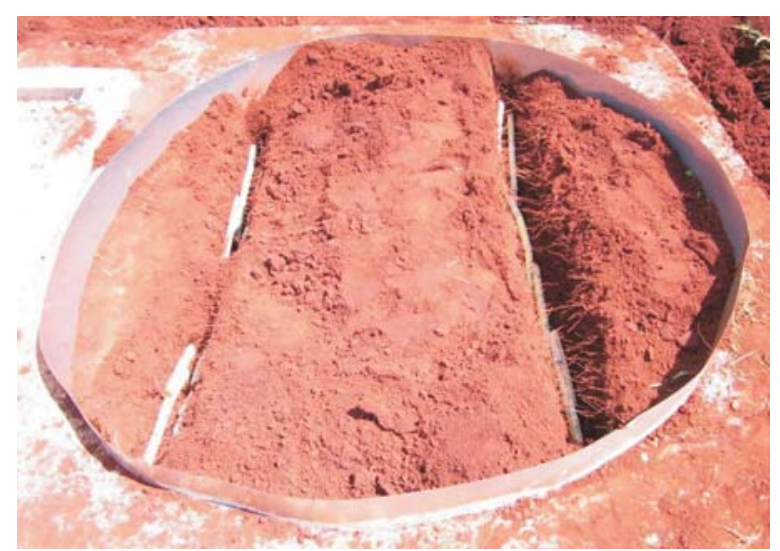

Figura 3 - Plantio da cana-de-açúcar.

$0,50 \mathrm{~m}$, promovendo $2,0 \mathrm{~m}$ de largura efetiva. $\mathrm{O}$ equipamento foi operado a $2,78 \mathrm{kgf} \mathrm{cm}^{-2}$ (40,0 psi), empregando água como diluente e volume de aplicação de $200 \mathrm{~L} \mathrm{ha}^{-1}$ ou $2 \mathrm{~L} 100 \mathrm{~m}^{-2}$ (calibração efetuada no local, em função da velocidade do aplicador em relação à área trabalhada). O preparo da calda foi de 2 litros para $100 \mathrm{~m}^{2}$. Assim, a aplicação de $400 \mathrm{~mL}$ de

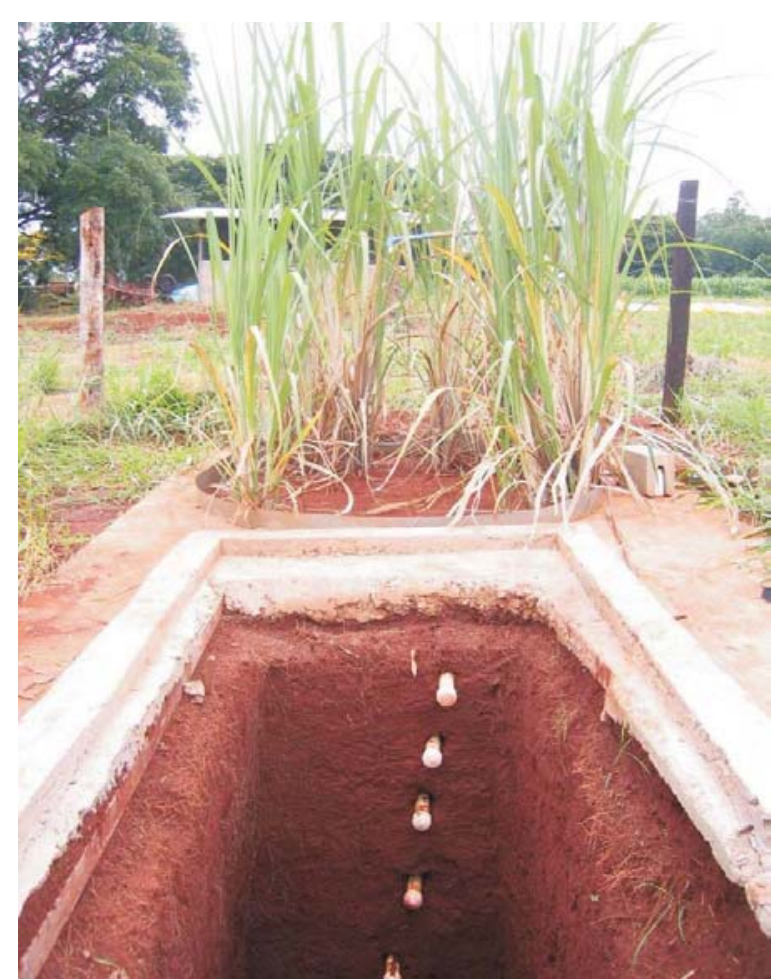

Figura 2 - Lisímetro montado no campo experimental da FEAGRI (janeiro 2007).

calda foi realizada numa área de $20 \mathrm{~m}^{2}$, incluindo a área do lisímetro $\left(3,14 \mathrm{~m}^{2}\right)$. O volume correspondente aplicado na área do lisimetro foi de $0,7536 \mathrm{~mL}$ ou $0,3768 \mathrm{~g}$ de i.a.

As amostras de água foram coletadas no dia seguinte às chuvas ocorridas no local. Para isso, as tampas de cada tubo foram desrosqueadas e a água contida em cada coletor foi retirada e armazenada em recipientes plásticos (Figura 4).

Em virtude da baixa ocorrência de chuvas nos meses posteriores à aplicação do herbicida (abril a setembro), instalou-se um sistema de irrigação no mês de julho. A irrigação foi feita no mês de agosto, em quatro aplicações, simulando as chuvas ocorridas no mês de janeiro do mesmo ano - por ter sido o mês de maior precipitação -, que totalizaram $255 \mathrm{~mm}$.

As amostras de água coletadas foram analisadas pelo Laboratório de Química Ambiental da Dow AgroSciences, em Mogi-Mirim-SP, credenciado pelo INMETRO em Boas Práticas de Laboratório (BPL). O método de análise utilizado 




Figura 4 - Coleta das amostras.

para detecção de resíduo de tebuthiuron na água de chuva foi Cromatografia Líquida de Alta Eficiência - CLAE.

\section{RESULTADOS E DISCUSSÃO}

A partir dos dados obtidos pela CLAE, obtiveram-se dois gráficos (Figuras 5 e 6), considerando, respectivamente, as relações entre concentração $\left(\mathrm{mg} \mathrm{kg}^{-1}\right)$ e massa (g) com a variável tempo, visando explicar o movimento vertical do herbicida tebuthiuron. Observando as curvas de tendência, nota-se que em um período aproximado de 180 dias não seria encontrado produto nas amostras de água de chuva, devido à sua possível degradação biológica ou retenção nas partículas de argila.

Os resultados obtidos no experimento que considera um solo indeformado, com a cultura da cana-de-açúcar implantada, em que a água recebida é direcionada no sentido vertical descendente, bem como as limitações do sis-

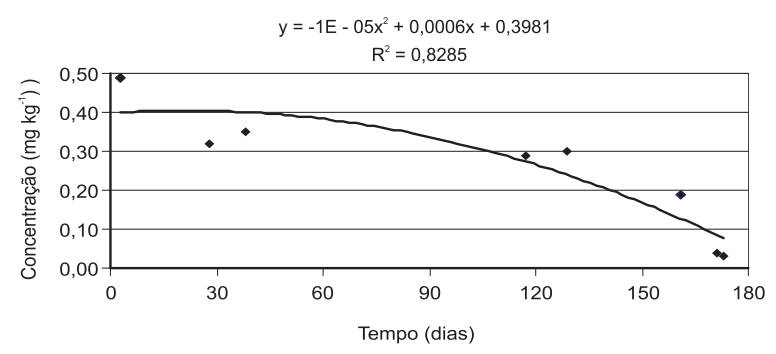

Figura 5 - Deslocamento do herbicida tebuthiuron concentração $\left(\mathrm{mg} \mathrm{kg}^{-1}\right) \mathrm{x}$ tempo (dias). tema lisímetro de drenagem modificado - mostraram que cerca de $24 \%$ do produto atingiu $30 \mathrm{~cm}$ de profundidade, correspondente ao primeiro coletor, e apenas $5,4 \%$ alcançou $52 \mathrm{~cm}$, correspondente ao segundo coletor, no período de 180 dias. Esse fato evidencia que mais de $70 \%$ do produto aplicado fica retido a menos de $30 \mathrm{~cm}$ de profundidade e/ou é degradado por meio de fatores, principalmente, bióticos do ambiente, visto que, de acordo com Rodrigues \& Almeida (2005), a degradação por meio de fatores abióticos é insignificante. Esses resultados corroboram aqueles encontrados por Eaton (1978), Lorenzi (1984), Blanco \& Lorenzi (1984) e Bovey et al. (1992), em que resíduos de tebuthiuron, em colunas de lixiviação, apresentaram pequena movimentação no solo, sendo encontrados em profundidades não superiores a $30 \mathrm{~cm}$, tanto em solos argilosos como em arenosos. Os resultados ainda estão de acordo com aqueles observados por Boeira \& Dornelas de Souza (2005), em que o movimento desse herbicida não ultrapassou 20 e $10 \mathrm{~cm}$ de profundidade, respectivamente, em solos de textura média e argilosa. Os resultados também confirmam aqueles verificados por Cerdeira et al. (2006), que coletaram amostras de solo (textura média e argilosa) em diferentes profundidades e não encontraram resíduos de tebuthiuron abaixo de $40 \mathrm{~cm}$.

Mesmo considerando as limitações do sistema experimental adotado, pode-se inferir que a dificuldade de lixiviação do herbicida, no perfil do solo, pode ter ocorrido devido à sua adsorção à matéria orgânica e à fração argila do solo, o que foi verificado nos trabalhos conduzidos por De Mello Filho et al. (1985), Matallo et al. (2003, 2005), Boeira \& Dornelas de Souza (2005) e Gomes et al. (2006) - sendo esse comportamento comum em solos com alta capaci-

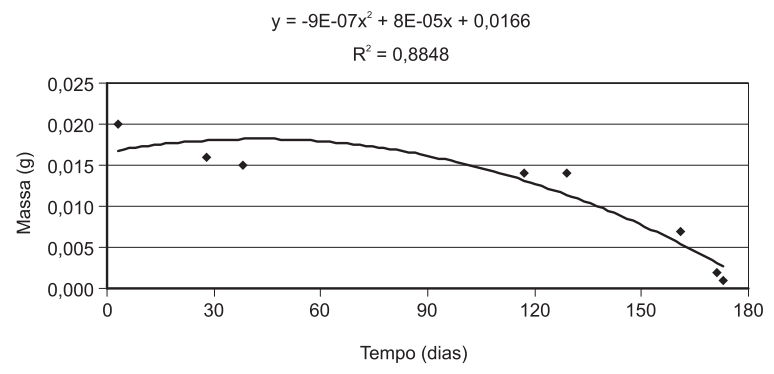

Figura 6 - Deslocamento do herbicida tebuthiuron - massa (g) $\mathrm{x}$ tempo (dias). 
dade de troca cationica (CTC) (Rodrigues \& Almeida, 2005) -, e/ ou ainda à ação, principalmente, de fatores bióticos, conforme comentado anteriormente.

Considerando as condições em que o experimento foi conduzido, admitindo-se a premissa de que solos argilosos apresentam alta CTC e que o teor de argila é um fator importante na baixa movimentação vertical do herbicida tebuthiuron - ressaltando ainda que a maioria dos solos do Estado de São Paulo onde se cultiva cana-de-açúcar são argilosos -, conclui-se, confirmando a hipótese testada, que o tebuthiuron apresenta baixa mobilidade no perfil do solo e, portanto, pequeno potencial de contaminação das águas subterrâneas em solo de textura característica para cultivo da cultura de cana-de-açúcar nesse Estado.

\section{LITERATURA CITADA}

ARMAS, E. D. et al. Uso de agrotóxicos em cana-de-açúcar na bacia do rio Corumbataí e o risco de poluição hídrica. Química Nova, v. 28, n. 6, p. 975-982, 2005.

BLANCO, H. G.; LORENZI, H. Persistência e resíduos de herbicidas em solo cultivado com cana-de-açúcar. Dados preliminares. In: CONGRESSO BRASILEIRO DE HERBICIDAS E PLANTAS DANINHAS, 15., 1984, Belo Horizonte. Resumos... Belo Horizonte: SBHED, 1984. p. 19.

BOEIRA, R. C.; DORNELAS DE SOUZA, M. Movimento do herbicida tebuthiuron no perfil de três tipos de solo através de colunas de lixiviação. Jaguariúna: Embrapa Meio Ambiente, 2005. 4 p. (Comunicado Técnico, 25)

BOVEY, R.; MEYER, R. E.; HEIN JR., H. Soil persistence of tebuthiuron in the claypan resource areas of Texas. Weed Sci., v. 30, n. 2, p. 140-144, 1992

CERDEIRA, A. L. et al. Fate of tebuthiuron herbicide in a recharge area of guarany aquifer in sugarcane field in Brazil. In: CONGRESSO BRASILEIRO DA CIÊNCIA DAS PLANTAS DANINHAS, 25., 2006. Brasília. Anais... Brasília: 2006. CD ROM.

COLETI, J.T. Técnica cultural de plantio. In: PARANHOS, S.B. (Coord.) Cana-de-açúcar cultivo e utilização Campinas: Fundação Cargill, v. 1, p. 271-283, 1987.

DE MELlO FILHO, A. T. de; ROCHA, C. L.; SILVA, S. A. da; HONDA, T. Estudo da lixiviação e degradação de tebuthiuron em solos dos estados de São Paulo e Alagoas. Stab, v. 2, p. 47-51, 1985.

DOW AGROSCIENCES. Manual de produtos. São Paulo: 2005.
DUARTE, N. F. Potenciais impactos ambientais da monocultura da cana-de-açúcar. In: VALADÃO, R. C.; LANDAU, E. C. (Eds.). Análise integrada do meio ambiente. Lagoa da Prata, Belo Horizonte, UFMG/PMLP. 2003. CD-ROM.

EATON, B. J. et al. Soil residues of tebuthiuron following single and repeat applications in sugarcane. In: ISSCT CONGRESS, 16., 1977, São Paulo. Proceedings... São Paulo: ISSCT, 1978. p.1049-1058

\section{EMPRESA BRASILEIRA DE PESQUISA}

AGROPECUÁRIA - EMBRAPA. Centro Nacional de Pesquisa de Solos. Sistema brasileiro de classificação de solos. Rio de Janeiro: 1999. 412 p

GOMES, M. A. F. Movimento do herbicida tebuthiuron em dois solos representativos das áreas de recarga do aqüífero Guarani. R. Bras. Eng. Agríc. Amb., v.10, n. 2, p. 479-483, 2006.

INOUE, M. H. et al. Critérios para avaliação do potencial de lixiviação dos herbicidas comercializados no estado do Paraná. Planta Daninha, v. 21, n. 2, p. 313-323, 2003.

LENG, M. L.; LEOVEY, E. M. K.; ZUBKOFF, P. L. Agrochemical environmental fate state of the art. New York: CRC Press Lewis Publishers, 2000. 410 p.

LORENZI, H. Comportamento dos resíduos de tebuthiuron em solos cultivados com cana-de-açúcar. In: SEMINÁRIO DE TECNOLOGIA AGRONÔMICA, 2., 1984, Piracicaba. Anais... Piracicaba: 1984. p. 263-272.

LOURENCETTI, C. et al. Avaliação do potencial de contaminação de águas subterrâneas por pesticidas: comparação entre métodos de previsão de lixiviação. Pesticidas: R. Ecotoxicol. Meio Amb., v. 15, n. 1, p. 1-14, 2005.

LUIZ, A. J. B.; NEVES, M. C.; DYNIA, J. F. Implicações potenciais na qualidade das águas subterrâneas das atividades agrícolas na Região Metropolitana de Campinas, SP. Jaguariúna: Embrapa Meio Ambiente, 2004 (Boletim de Pesquisa e Desenvolvimento, 25)

OLIVEIRA, J. B.; CAMARGO, M. N.; CALDERANO FILHO, B. Mapa pedológico do Estado de São Paulo: legenda expandida. Campinas: Instituto Agronômico; Rio de Janeiro: Embrapa-Solos, 1999. 64 p.

MATALLO, M. B. et al. Lixiviação dos herbicidas tebuthiuron e diuron em colunas de solo. Pesticidas: R. Ecotoxicol. Meio Amb., v. 13, n. 1, p. 83-90, 2003.

MATALLO, M. B. et al. Sorption, degradation and leaching of tebuthiuron and diuron in soil columns. J. Environ. Sci. Health, v. 40, n. 1, p. 39-43, 2005. 
PESSOA, M. C. P. Y. et al. Identificação de áreas de exposição ao risco de contaminação de águas subterrâneas pelos herbicidas atrazina, diuron e tebuthiuron. Pesticidas: R. Ecotoxicol. Meio Amb., v. 13, jan./dez., p. 111-122. 2003

PROCÓPIO, S. O. et al. Manejo de plantas daninhas na cultura da cana-de-açúcar. Viçosa, MG: Universidade Federal de Viçosa, 2003. 150 p.
RODRIGUES, B. N.; ALMEIDA, F. S. Guia de herbicidas. 5. ed. Londrina: Grafmarke, 1995. $592 \mathrm{p}$

SPADOTTO, C. A. et al. Monitoramento do risco ambiental de agrotóxicos: princípios e recomendações Jaguariúna: Embrapa Meio Ambiente, 2004. 29 p. (Documento, 42) 